\section{Coupled, but not conjugated, in cancer}

\section{By Michael J. Haas, Senior Writer}

California researchers have shown that a known tumor-penetrating peptide called iRGD can boost the efficacy of cancer drugs without having to be conjugated to them-a laborious step that can reduce a drug's potency. ${ }^{1}$ By demonstrating that conjugate synthesis is no longer necessary, the team has made its peptide amenable to use with a wider range of cancer drugs.

The group previously showed that the peptide's arginine-glycineaspartic acid (RGD) motif bound to integrin $\alpha_{\mathrm{V}}$ (CD51), a target that is overexpressed on tumor vasculature compared with its expression on normal vasculature. As a result, cancer drugs conjugated to internalizing RGD (iRGD) had greater uptake into tumors than unconjugated therapeutics. ${ }^{2}$

Now, the group has taken conjugation out of the equation.

Mice injected with unconjugated iRGD and either doxorubicin, liposomal doxorubicin, Herceptin trastuzumab or Abraxane nabpaclitaxel had greater drug accumulation in the tumor by up to 40 -fold than mice injected with one of the drugs alone. The result was greater reductions in tumor growth.

The drug-peptide combination was as effective as threefold higher doses of drug alone.

The team also found that iRGD increased uptake of iron oxide- and phage-based nanoparticles by prostate tumors in mice.

Finally, the researchers showed that organs from mice co-treated with drug and peptide had no macroscopic metastases. Those results demonstrated that increasing the permeability of tumor vasculature to cancer drugs did not enhance the ability of tumor cells to migrate and metastasize, team leader Erkki Ruoslahti told SciBX.

Ruoslahti is professor of molecular, cellular and developmental biology at the University of California, Santa Barbara (UCSB) and the Sanford-Burnham Medical Research Institute.

In addition to researchers at UCSB and Sanford-Burnham, the team included Daniel Greenwald, an oncologist at the Cancer Center of Santa Barbara. Data were reported in Science.

"Not having to synthesize, test and administer a drug-peptide conjugate would offer flexibility and ease in harnessing the peptide's therapeutic value" in improving drug delivery to tumors, said Lance Leopold, CMO and VP of clinical development at cancer company
Ascenta Therapeutics Inc.

Ascenta's AT-101, a small molecule pan-inhibitor of the B-cell lymphoma 2 (BCL-2; BCL2) family of proteins, is in Phase II testing to treat progressive or recurrent glioblastoma multiforme (GBM) and other cancers.

"I would call this work revolutionary," said Emma Shtivelman, director of cancer research at Bionovo Inc. "It holds a promise of greatly improved delivery of various classes of anticancer drugs selectively to tumors. Obviously, this will have profound implications for patients' quality of life by reducing side effects associated with most anticancer drugs."

Bionovo's Bezielle (BZL101), an aqueous extract from the herb Scutellaria barbata that inhibits glycolysis, is in Phase II testing to treat advanced breast cancer and Phase I/II testing to treat pancreatic cancer.

\section{Permeability permutations}

Although the treated mice showed no hints of metastasis, Shtivelman told SciBX the potential pitfalls of increased tumor permeability need to be further explored. The models used by Ruoslahti's team could not adequately predict the risk of metastasis associated with iRGD treatment in humans, she said, because "the growth and metastases of the majority of human cancers occurs within a time longer than the few weeks" needed to grow xenograft tumors in mice.

Shtivelman added that spontaneous metastases of human tumor cells are unlikely to occur in conventional xenograft mouse models. Mouse models in which human tumor cells grow in human tissue grafts would be more relevant for examining iRGD's effects on human vasculature and its potential to increase tumor cell metastasis, she said.

Leopold also noted that iRGD could have the usual drawbacks associated with peptide therapeutics, including a short in vivo half-life and the potential for immunogenicity.

Despite her safety concerns, Shtivelman was sanguine about iRGD's therapeutic potential and wanted to see the peptide moved into Phase I trials. "The possibility of greatly improving delivery of various anticancer drugs is just too exciting not to explore in patients," she said. "Any clinical protocol that uses drugs such as doxorubicin or taxol could be modified to incorporate combination treatment with iRGD."

Leopold thinks testing iRGD in combination with approved cancer therapies would be a good first step. "Though theoretically of interest, testing multiple unapproved agents is problematic," he said.

Shtivelman nevertheless wanted to know whether the free peptide also could help deliver small interfering RNAs to tumors. "Delivery of modified and/or conjugated siRNAs is a major obstacle" to tapping the therapeutic potential of siRNA, she noted.

Indeed, Ruoslahti said the team's ongoing work includes testing the iRGD peptide in combination with therapeutic siRNAs and with orally delivered drugs in models of breast and prostate cancer. 


\section{ANALYSIS}

Although the team does not expect to study the peptide in a wide range of cancers, "we do plan to test the treatment in models of pancreatic adenocarcinoma because these tumors are so difficult to treat in patients," Ruoslahti said. For those studies, the team intends to combine the peptide with Eli Lilly and Co.'s Gemzar gemcitabine or with experimental antibodies.

He said the team has conducted additional studies to demonstrate that the peptide does not increase tumor metastasis and will report those data in a forthcoming publication.

According to Ruoslahti, Sanford-Burnham holds several issued patents that cover the findings reported in Science and is interested in licensing or partnering arrangements.

Roche's Genentech Inc. unit markets Herceptin, a humanized mAb against HER2 (ERBB2; neu), to treat breast cancer.

Abraxis BioScience Inc. and Otsuka Pharmaceutical Co. Ltd. market Abraxane, an albumin-stabilized nanoparticle formulation of paclitaxel, to treat breast cancer.
Haas, M.J. SciBX 3(17); doi:10.1038/scibx.2010.514

Published online April 29, 2010

\section{REFERENCES}

1. Sugahara, K. et al. Science; published online April 8, 2010; doi:10.1126/science.1183057

Contact: Erkki Ruoslahti, University of California, Santa Barbara, Calif. e-mail: ruoslahti@burnham.org

2. Sugahara, K. et al. Cancer Cell 16, 510-520 (2009)

COMPANIES AND INSTITUTIONS MENTIONED

Abraxis BioScience Inc. (NASDAQ:ABIII), Los Angeles, Calif. Ascenta Therapeutics Inc., Malvern, $\mathrm{Pa}$.

Bionovo Inc. (NASDAQ:BNVI), Emeryville, Calif.

Cancer Center of Santa Barbara, Santa Barbara, Calif.

Eli Lilly and Co. (NYSE:LLY), Indianapolis, Ind.

Genentech Inc., South San Francisco, Calif.

Otsuka Pharmaceutical Co. Ltd. (Tokyo:4768), Tokyo, Japan

Roche (SIX:ROG; OTCQX:RHHBY), Basel, Switzerland

Sanford-Burnham Medical Research Institute, La Jolla, Calif. University of California, Santa Barbara, Calif. 